\title{
Entanglement Distribution in Optical Networks
}

\author{
Alex Ciurana, Vicente Martin, Jesus Martinez-Mateo, Bernhard Schrenk, Member, IEEE, \\ Momtchil Peev, and Andreas Poppe
}

\begin{abstract}
The ability to generate entangled photon pairs over a broad wavelength range opens the door to the simultaneous distribution of entanglement to multiple users in a network by using centralized sources and flexible wavelength-division multiplexing schemes. Here, we show the design of a metropolitan optical network consisting of tree-type access networks, whereby entangled photon pairs are distributed to any pair of users, independent of their location. The network is constructed employing commercial off-the-shelf components and uses the existing infrastructure, which allows for moderate deployment costs. We further develop a channel plan and a network-architecture design to provide a direct optical path between any pair of users; thus, allowing classical and one-way quantum communication, as well as entanglement distribution. This allows the simultaneous operation of multiple quantum information technologies. Finally, we present a more flexible backbone architecture that pushes away the load limitations of the original network design by extending its reach, number of users and capabilities.
\end{abstract}

\section{INTRODUCTION}

$\mathbf{E}$ NTANGLEMENT is one of the most striking features of the quantum world. This property, with no classical counterpart, can be used in many applications of quantum information processing, such as the well-known quantum key distribution (QKD) [1], superdense coding [2] or quantum teleportation [3], among others. All these applications require quantum and classical communications, thus needing two communication channels: a quantum channel and a conventional one. The former, governed by the laws of quantum mechanics, is used to exchange quantum states (typically photons) carrying quantum information while the latter is assumed to be an error-free channel. Further requirements can be imposed on the classical channel for certain purposes (e.g., it must be authentic if it is used to extract a secret key in QKD post-processing).

A. Ciurana, V. Martin, and J. Martinez-Mateo are with the Facultad de Informática, Universidad Politécnica de Madrid, 28040 Madrid, Spain (e-mail: aciurana@fi.upm.es; vicente@fi.upm.es; jmartinez@fi.upm.es).

B. Schrenk, M. Peev, and A. Poppe are with the business unit Optical Quantum Technology, Safety \& Security Department, Austrian Institute of Technology, A-1220 Vienna, Austria (e-mail: Bernhard.Schrenk@ait.ac.at; Momtchi1.Peev@ait.ac.at; Andreas.Poppe@ait.ac.at).
These applications of quantum information processing, in general, and entanglement, in particular, require point-to-point communications as well as a high degree of isolation from the environment to avoid decoherence and to enable noise-free quantum channel operation to allow detecting the weak quantum signals. Therefore, the simplest way to design a network supporting quantum channels is to use a point-to-point topology with dedicated links (typically optical fibers). Unfortunately, this is a very expensive and therefore, impractical solution. Using existing infrastructure and sharing it with other users of the communication channels (quantum or conventional ones) would reduce the deployment and operational costs to an acceptable level.

For this reason, the study of the integration of quantum signals in point-to-point links via multiplexing [4]-[8] or using dark fibers in the field [9]-[13] has been a long standing topic in quantum communications. As a next logical step, a point-tomultipoint network scenario such as the one provided by access networks in standard optical communication networks should be considered. In the non-entangled case, there have been proposals for access networks based on time division multiplexing (TDM) technologies [14]-[20] and wavelength division multiplexing (WDM) [19], [21]. For entangled pairs of photons, only WDM-based access networks have been studied [22]-[25]. Beyond these approaches, the next step has been to devise the integration of any-to-any quantum communications in architectures for metropolitan-wide networks [14], [26]-[29] that lead to an increase of the number of quantum users, capacity and reach. In particular, because of the high cost of these infrastructures, we consider the quantum metropolitan optical network (MON) presented in [27] as a starting point for a framework to allow entanglement distribution together with other quantum information technologies.

The goal of this work is to replace the dedicated optical fiber used as a quantum channel between the source of entangled photon pairs and two users by a flexible switched fiber network. The presented practical MON designs support entanglement distribution AND one-way quantum communication to any pair of users in this infrastructure. Additionally we further extend the network functionality to accommodate classical classical communication between the users by using wavelength multiplexing of the latter with quantum channels over the same fiber. Hence, the network provides all basic transmission resources needed by quantum information technologies, thus sharing the cost among many possible users. To be practical, it must integrate well with the deployed infrastructure and be based on standard, readily available telecom-class components designed for mass deployment. This optical transport layer requires a 


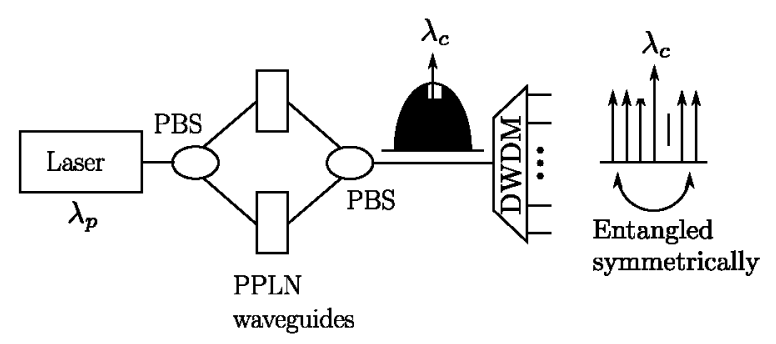

Fig. 1. Scheme and output of a broadband source of entangled photon-pairs (for details see [25]). A laser pumps the PPLN waveguides. SPDC generate photon pairs over a broad spectrum that is sliced into DWDM channels using a demultiplexer. Hence, DWDM channels are entangled symmetrically. PBS stands for polarizing beam splitter.

fast turn-on time of the QKD system to stabilize the quantum channel (e.g., the polarization) and synchronize the peers to a common time frame. In the case of polarization entanglement QKD this operational functionality has been demonstrated in principle [30], [31] but would need to be optimized further for smooth system integration. On the other hand, QKD security is fully independent from the optical transport layer. Adding optical components would only lead to loss, i.e. in the entanglement case, to a decrease the quality of entanglement (e.g, depolarization), which in turn results in a corresponding decrease of the amount of secure key generated by QKD. It is worth mentioning that the optical network does not solve any security constraints related to the authentication of the classical communication (the amount of needed pre-shared secret is not reduced) nor does it open a security loophole in this respect.

The rest of this paper is organized as follows. First, in Section II we describe the operation mode of the entanglement source considered in our work, and the canonical architecture of a in Section III. In Section IV we propose a MON, in which only entangled quantum signals are distributed. Later, in Section V, we present a channel plan that transmits quantum, one-way and entangled, and conventional signals. Based on this channel plan, we design two networks employing different architectures in Sections VI and VII, respectively. Finally, we discuss both architectures and draw some conclusions in Section VIII.

\section{BroAdband Source of ENTANGLEd PAirs of Photons}

The scheme of a broadband source generating entangled photon pairs is shown in Fig. 1 and its output is based on a spontaneous parametric down conversion (SPDC) process. A narrow-band continuous wave laser diode at $\lambda_{p}$ pumps the periodically-poled lithium niobate (PPLN) waveguides to obtain degenerate photons with a spectrum centered at $\lambda_{c}=2 * \lambda_{p}$. A photon at the lower part of the spectrum is frequency correlated with a one of the upper part and both are polarization entangled each with the other. The wavelength $\lambda_{p}$ is selected such that the pairs are generated into (the spectral slots of) dense wavelengthdivision multiplexing (DWDM) channels. Note that the signal produced by the source has constant spectral density, therefore using a denser DWDM grid in order to connect more users is in a trade-off with a lower photon rate per channel. The broadband output makes these sources especially suitable for use in

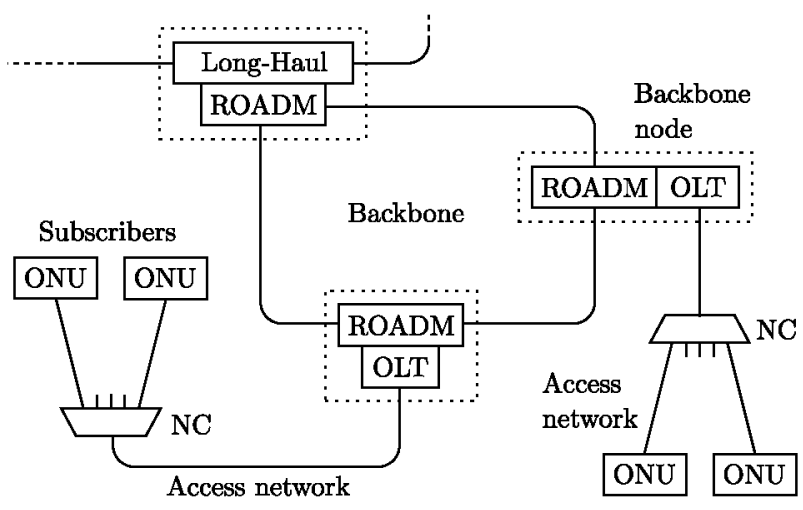

Fig. 2. Architecture of a canonical MON. Users are connected to tree-type access network via a NC. All the access networks are connected to a ring-shaped or mesh backbone network via an OLT and a reconfigurable optical add-drop module.

DWDM-based networks, since a single source can serve many users simultaneously.

As a result, photons of each DWDM channel are entangled with the ones in a corresponding other channel. This is of special importance for the network, in which we want to route a couple of entangled DWDM channels from the source to a pair of users. Selecting one of the DWDM channels fixes the second one that has to be used. Note that, when such source is switched on, the entire broadband signal is always produced. We cannot produce entangled photon-pairs only at certain wavelengths within the output spectrum. Thus, in order to control which pairs are distributed, we need to use network components (NCs) at the output of the source (e.g., switches).

In particular, we consider a source as in [25] with the following characteristics: (i) $\lambda_{p}=775 \pm 5 \mathrm{~nm}$, (ii) $\lambda_{c}$ in the $\mathrm{C}$ band and (iii) with a spectral width of $70 \mathrm{~nm}$. Based on the same scheme, sources using shorter PPLN waveguides or chirped poling structures [32] could generate entangled photon pairs over a spectral width of the entire S-C-L-telecom band. In terms of output power, the source generates $4.5 \times 10^{5} \mathrm{pairs} / \mathrm{s} / \mathrm{mW} / \mathrm{GHz}$. For waveguide-based sources typically pumping powers below $1 \mathrm{~mW}$ are needed not to be limited by unwanted multi-pair effects [33].

\section{METROPOLITAN OPTICAL NETWORKS}

The architecture of a canonical MON [34] is depicted in Fig. 2. In the figure, tree-type access networks based on passive optical network technology are connected to a core or backbone network. This architecture allows users to communicate with other users within the same access network, or in a different access network through the backbone.

End users (i.e., subscribers) in the access networks are connected to optical network units which are then connected by a NC. Their signals are multiplexed using TDM or WDM, respectively. In the TDM case, the NC is an optical splitter, while in the WDM case it is a dense WDM (DWDM) multiplexer based on arrayed waveguide grating (AWG) technology. The $\mathrm{NC}$ is then connected to an optical line terminal (OLT), located at the premises of the service provider. The OLT coordinates 
TABLE I

INSERTION LOSSES

\begin{tabular}{lcc}
\hline \hline & $\begin{array}{c}\text { Operating } \\
\text { wavelength } \\
\text { range (nm) }\end{array}$ & \\
Component & 1550 & Insertion loss (dB) \\
\hline Single-mode fibre ITU-T G.652 & 1310 & 0.2 per km \\
Single-mode fibre ITU-T G.652 & $1260-1610$ & 0.32 per km \\
$1: 2$ Splitter 50/50 & $1260-1610$ & 3.6 \\
$1: 2$ Splitter 10/90 & $1260-1610$ & $11.3 / 0.5$ \\
$1: 32$ Splitter & $1270-1610$ & 16.5 \\
One-channel CWDM filter & $1270-1610$ & 0.4 (ref.), 0.6 (fil.) \\
Four-channels CWDM mux & $1270-1610$ & 1 \\
Eight-channels CWDM mux & $1260-1360$ & 1.5 \\
$1310 / 1550$ WDM mux & $\& 1500-1600$ & 0.5 \\
Circulator & & 0.8 \\
32 -channels AWG (100 GHz) & $1533-1558$ & 3 \\
$4 \times 4$ to $192 \times 192$ Switch & $1270-1675$ & 1 \\
\hline \hline
\end{tabular}

the traffic within the access network and performs the necessary conversions to introduce the signal in the backbone network.

Usually, the backbone network topology is either a ring (as in Fig. 2) or a mesh, and coarse WDM (CWDM) is the most common technology used by carriers to multiplex its traffic. The network consists of multiple backbone nodes that switch signals (depending on their wavelength) between the backbone and the access networks. For this purpose, an optical add-drop multiplexer $(O A D M)^{1}$ is connected to the OLT. In our network, both devices, OADM and OLT, are considered to be situated in the same location, which allows us to propose a backbone node that does not interrupt the quantum signals by performing, for example, electro-optical conversions.

In this work we are looking for simple deployment and maintenance, hence we will stick to the presented typical technologies for the design of quantum MONs. In particular, we propose CWDM [35] for the backbone and DWDM [36] for the access networks. This allows to use common commercial components. Note that in contrast to TDM-based networks, the proposed one does not require any synchronization and allows for simultaneous communications. Hereafter we use $C X$ and $D X$ to refer to the CWDM channel with $\lambda=X \mathrm{~nm}$ and the DWDM channel with $\lambda=X \mathrm{~nm}$, respectively. Further, whenever required for the calculations, we will consider the $100 \mathrm{GHz}$ DWDM ITU-T G.694.1 grid and 32-channels AWGs. The fiber distances in the network are assumed to be: $1 \mathrm{~km}$ between users and the $A W G$, $3.5 \mathrm{~km}$ between the AWG and a backbone node, and $4 \mathrm{~km}$ between neighboring backbone nodes; corresponding to our experimental testbed. Insertion losses of common NCs [37]-[40] are shown in Table I.

The aim of this work is to design a MON, based on the above mentioned technologies, and a design of the backbone nodes that can enable the routing of entangled pairs of photons between any two network users. In addition to routing, the overall design must take into account other aspects such as the maximum number of users that can be simultaneously connected to the network,

\footnotetext{
${ }^{1}$ Also common in its reconfigurable version, i.e., reconfigurable OADM or ROADM.
}

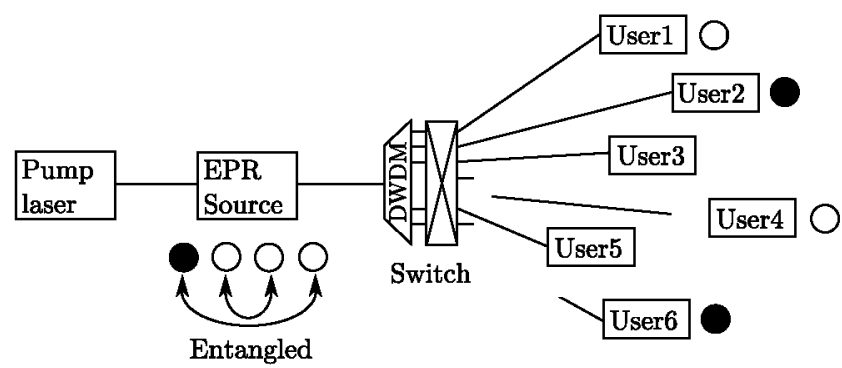

Fig. 3. Broadband entanglement source connected to the users via a switch, which is necessary to enable all possible user pairings. This is equivalent to a WDM-based access network.

as well as the loss budget (that, for instance, directly affects the maximum distance and throughput at which two users can be connected)

\section{ENTANGLEMENT-ONLY MON}

We start by proposing a design for MONs supporting only entanglement distribution. Despite its simplicity, this design presents an advance over the state-of-the-art of entanglementonly telecom optical networks [22]-[25] and also serves the purpose to illustrate the operation mode of the sources and their integration in WDM-based networks.

\section{A. Single Access Network}

The first approach is to directly connect the users to the output of the source. This is shown in Fig 3, including an intermediate switch that is required to change among all possible user pairings. The use of switches in optical networks supporting quantum communications has been already studied [41]. The number of input and output channels of the switch must be matched to the available number of DWDM-channels of the source and the number of users, respectively. The resulting point-to-multipoint network is a WDM-based access network.

The number of established connections at a given time is limited by the bandwidth of the source. More users can be connected physically to the switch to receive available photons on demand. With this simple solution only users attached to a central point of a star network could be connected.

\section{B. Two Access Networks}

A more flexible network can be obtained by adding a backbone network to combine the simple access network from above. Thereby the architecture changes due to the shift of the source to the backbone. Let us assume now that all DWDM channels from the access network are contiguous. We can group a set of them within the width of a single ITU-T G.694.2 CWDM channel. Therefore, since the spectrum of the source is wider than a CWDM channel and by choosing the pump wavelength accordingly, we can generate entangled photon-pairs situated in DWDM channels corresponding to two different CWDM channels. If we send different CWDM channels to different access networks, we can have entanglement shared between two access networks. 




Fig. 4. Broadband entanglement-source serving two access networks. The output of the source is demultiplexed in DWDM channels and grouped in CWDM channels 1510 and 1550. Each CWDM channel is dropped by an OADM in a different access network.

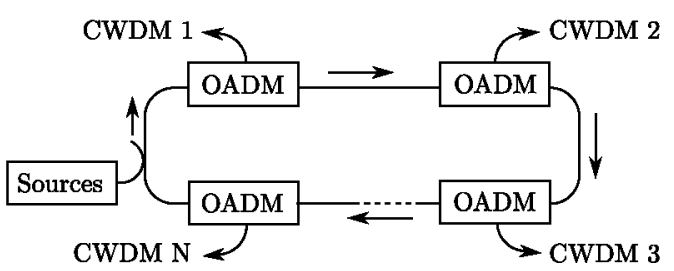

Fig. 5. Design of a entanglement-only MON with $N$ access networks and a ring-shaped passive backbone. Each access network has assigned a CWDM channel. Sources, connected to the backbone, distribute entanglement over all single CWDM channels and possible pairs, i.e., all single access networks and pairs of them.

To do so, at the source, we select the DWDM channels belonging to the chosen CWDM channels (i.e., access networks) and we multiplex them into a single optical fiber. In the optical fiber we add two CWDM OADMs to drop the corresponding CWDM channels at the corresponding access network ${ }^{2}$. The result is a backbone network with an open-ring topology that can be built retaining passive technology. The network is shown in Fig. 4. The source is directly connected to the backbone and distributes entanglement between C1510 and C1550 (blue and orange in the figure, respectively). Depending on the particular CWDM channels used, the source configuration will change accordingly by choosing the appropriate multiplexers to group the DWDM channels used.

\section{Metropolitan Optical Network}

Using these two subnetworks as basic blocks, we can build an entanglement-only MON with $N$ access networks (see Fig. 5). For example, given $N=3$, we need to address 3 single access networks as in Fig. 3, and $\left(\begin{array}{l}3 \\ 2\end{array}\right)=3$ pairs of access networks as in Fig. 4. Therefore, it requires a maximum of six sources: three of them to distribute entanglement in each access network, and another three to distribute entanglement between the possible three pairings of access networks. In this way, any user can share entanglement with any other one. Although the number

\footnotetext{
${ }^{2}$ Actually, note that the add function of the OADM is not used in these entanglement-only networks. Hence, the OADMs can be replaced by simple one-channel CWDM filters, which have fewer losses.
}

of sources increases quadratically with $N$ (namely as $\frac{N(N+1)}{2}$, the number of access networks is severely limited by the loss budget and the available spectrum. Moreover, the actual number of sources can be smaller since some of those combinations could be provided by a single source. For instance, a source that distributes entanglement between C1530 and C1570, can distribute it at the same time to the users in a single access network, e.g., C1550. Regarding the physical allocation of the sources, all of them are grouped as a single source and connected to the backbone.

Subsequently, a CWDM channel is assigned to each access network and backbone nodes route these channels to the different access networks. Finally, once within the access network, the AWG slices the CWDM channel into DWDM channels and a switch routes them to the appropriate user. Therefore, a DWDM channel is assigned to each AWG port in the access network thanks to AWG's periodicity ${ }^{3}$. Note that, when emitting a photon (from a pair) using a particular wavelength, the emitter is actually selecting the target/final access network and the AWG port.

However, in this network architecture, multiple sources might try to reach the same access network and thus to use the same CWDM channel. This is a consequence of the goal to provide entanglement distribution between each pair of users in each access network as well as between any user in this network an any other one in any of the remaining $N-1$ access networks. Hence, we need to carefully connect the sources when grouping them. We propose three solutions (see Fig. 6). In the first scheme, sources will use the full CWDM channel and a switch will decide which source uses that CWDM channel at each moment. In the second scheme, switches are moved nearer to the sources in order to decide which source uses each DWDM channel of each CWDM channel. Therefore, a CWDM channel is used by multiple sources at the same time. However, this flexibility comes at the cost of using a larger number of switches. In the third scheme, we remove all switches. Instead, we simply distribute the available DWDM channels among the sources. Hence, each source always uses the same DWDM channels.

Now, we calculate the maximum number of users that this network can support using the previously mentioned considerations (see Section III). First, the maximum number of access networks $N$ is established by the insertion losses. Note that, when using entangled photon-pairs, the losses are the sum of the two paths. Therefore, the worst path in terms of losses in our network is the sum of the two worst paths: from the source to access network $N-1$, and from the source to access network $N$. Considering a loss budget of $30 \mathrm{~dB}$ per entanglement-based link, we estimate $N=8$ : (i) $14.7 \mathrm{~dB}$ from the source to a user in the access network number $N=8$, (ii) $13.5 \mathrm{~dB}$ from the source to a user in the access network number $N-1$, and (iii) $13.5+14.7=28.2 \mathrm{~dB}$ overall loss for the link. As a result of the fixed mapping of CWDM channels, a maximum of eight CWDM channels are needed. Nevertheless, to reach this upper

\footnotetext{
${ }^{3}$ To simplify matters, we assume that a CWDM channel equals an AWG's spectrum band. However, this may not be the case and then some DWDM channels will be unusable.
} 


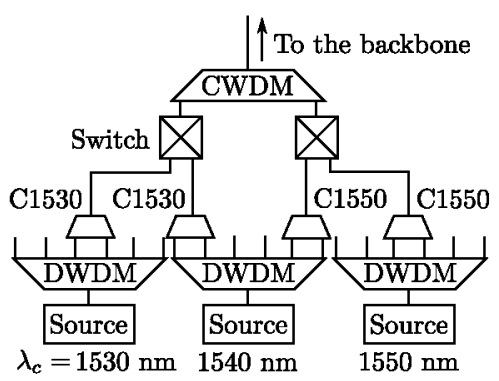

(a)



(b)

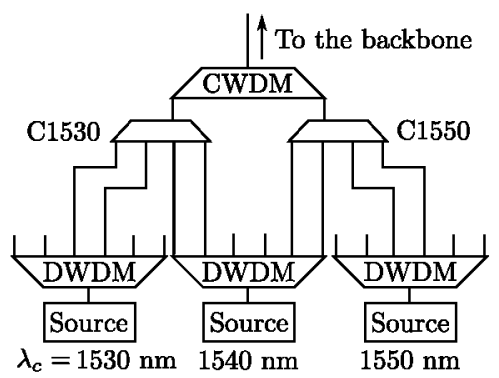

(c)

Fig. 6. Possible connection schemes for the entanglement sources in an entanglement-only MON with two access networks. In (a), switches are used to decide which source uses each CWDM channel. In (b), switches decide which source uses each DWDM channel; hence, a CWDM channel is used by multiple sources at the same time. In (c), CWDM channels are also shared among all sources but in a fixed way; a source has assigned always the same DWDM channels. (a) Connection scheme 1. (b) Connection scheme 2. (c) Connection scheme 3 .

bound, we need a source able to produce entangled photon-pairs between CWDM 1 and CWDM 8, this is, approximately $160 \mathrm{~nm}$ of overall band-width. Hence, depending on the spectral width of the sources used, the actual number of access networks can be lower. Finally, we can estimate the number of users by multiplying $N$ by the number of DWDM channels per CWDM channel (passband of approximately $13 \mathrm{~nm}$ ), e.g., $8 \cdot\lfloor 13 / 0.8\rfloor=128$ users with a $100 \mathrm{GHz}$ DWDM ITU grid. A higher loss budget would increase the maximum number of users by allowing more backbone nodes and more users per access network (AWGs with denser DWDM grids).

\section{ENABLING ONE-WAY CONVENTIONAL AND QUANTUM COMMUNiCATIONS OVER THE SAME FIBER INFRASTRUCTURE}

Up to now, the network has been designed to distribute only entangled photon pairs. As it was mentioned before, the large infrastructure cost of the network is the most important factor preventing the widespread adoption of quantum networks. Hence, in order to share costs and increase the chances of having a large-scale implementation, it makes sense to try to include as many communication capabilities as possible. In this spirit, we will discuss now how to incorporate direct communications, quantum and conventional, between users. The former will allow to use a higher variety of quantum information technologies, whereas the latter ones are essential to perform any conventional communication, either required by the quantum information protocols themselves or by external applications.

For this, let us start by adding the conventional signals to the network. As in [27], we will use two spectral bands separated by approximately $150 \mathrm{~nm}$ : the $O$ band $(1260-1360 \mathrm{~nm})$, and the $\mathrm{C}$ band and its vicinity $(1500-1600 \mathrm{~nm})$. This separation allows to effectively filter most of the noise and to reduce the crosstalk from conventional to quantum signals [16]; thus increasing the number of simultaneous signals that can be transmitted in the network [27]. The size of the bands is delimited by their separation and the operating wavelength range of the NCs (see Table I).

Based on the source characteristics, we assign the $O$ band to the conventional signals and the $\mathrm{C}$ band, and its spectral vicinity, to the quantum ones. The separation between bands guarantees that the spontaneous stokes Raman scattering from the conventional signal does not spoil the quantum signals [42]. Each band is then divided into CWDM channels and a pair of them, one in the $\mathrm{C}$ band and the other in the corresponding AWG period in the $\mathrm{O}$ band, is assigned to each access network. The operation mode of the backbone nodes and the AWGs at the access networks remains the same. Therefore, now each AWG port has assigned two DWDM channels: one containing single photons that are entangled with those in another DWDM channel in the $\mathrm{C}$ band, and another one, in the $\mathrm{O}$ band, containing conventional optical pulses. Note that both should be separated at the receiver using a filter.

For the connection of the sources, we use the scheme depicted in Fig. 6(c). Since DWDM channels are assigned and fixed to a specific source, each source is independent of the rest. As long as DWDM channels are distributed over the sources correctly (e.g., a DWDM channel is not assigned to more than one source), sources can be separated and connected at different points of the backbone network. In addition, by assigning and fixing a dedicated source to a subset of CWDM channels, we avoid wavelength-tuning of the source on-the-fly, a complex task that would affect all users receiving from that source.

So far and in contrast to [27], CWDM channels are shared among all sources, without leaving room for one-way quantum signals. We could use a third spectral band for one-way quantum signals. However, we are already occupying both lowattenuation telecommunication windows, and to use a third CWDM channel per access network would also increase the losses at the backbone node (as more components would be needed for the OADM). Therefore, we propose to share the quantum band between one-way single photon pulses and entangled pairs. To this end, among the DWDM channels assigned to each source, only a set of them will actually be connected to 


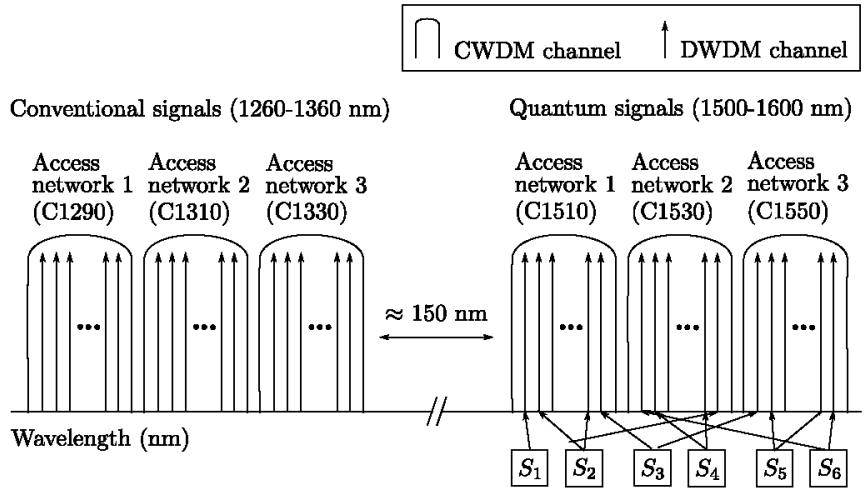

Fig. 7. Channel plan for a quantum MON. Each access network has assigned two CWDM channels, for quantum and conventional signals. They are spectrally separated to avoid any crosstalk, but belong to the same period of the AWG thus guaranteeing that they come out through the same physical port. Within the CWDM channel, DWDM channels are used for one-way communications or entanglement distribution. The figure shows how entanglement sources $\left(S_{x}\right)$ are arranged to ensure entanglement among any pair of users in the network. The black arrows in the quantum part of the spectrum are left free for one-way quantum signals.

the source. The rest are left free for one-way quantum signals ${ }^{4}$. Furthermore, this set of channels is fixed. Instead of reconfiguring the source, we use the switch at the access network to connect the user to the corresponding AWG port.

The resulting channel plan is shown in Fig. 7. The figure shows the arrangement of bands, CWDM and DWDM channels. Colored arrows indicate DWDM channels used by entanglement sources $\left(S_{x}\right)$. In this case, using six sources we can distribute entanglement to any pair of users of the network, within the same access or in separate ones. The rest of DWDM channels (in black) are available for one-way signals. Users can connect to each type configuring the switches at the access networks.

A substantial advantage of our design is its scalability when facing a demand increase of entangled channels. We can gradually provide more channels by just connecting the outputs of the source to the DWDM multiplexer. Obviously, this is done at the expense of the available channels for one-way quantum communications.

\section{Quantum MON With ENTANGLEMENT Distribution}

Here we modify the previous network design in order to use more sources of entangled photon pairs in the new channel plan. As before, we will stick to passive optical technology for the backbone nodes, which makes them static from the routing point of view, i.e., signals are always routed in the same way.

\section{A. Backbone Node}

A design of the backbone node is provided in Fig. 8. It is based on a typical OADM and can be built out of off-the-shelf components. It is responsible for routing CWDM channels to access networks and for adding the source's signal as well as

\footnotetext{
${ }^{4}$ And vice versa; one-way systems must not emit quantum signals at the DWDM channels reserved for entanglement distribution.
}

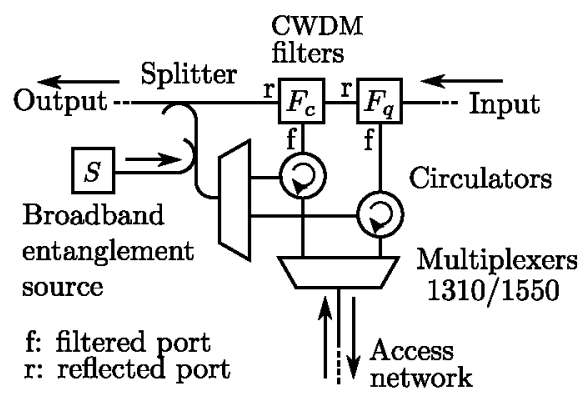

Fig. 8. Design of a passive backbone node for a ring-shaped backbone that includes a broadband source of entangled photon-pairs.

TABLE II

Losses of the Passive Backbone NoDe With ENTANGLEMENT-CAPABILITY DEPICTED IN FIG. 8

\begin{tabular}{lccc}
\hline \hline Action & Losses Conv. & Losses Quantum & Losses Ent. \\
\hline Add & $5.9 \mathrm{~dB}$ & $5.9 \mathrm{~dB}$ & $3.6 \mathrm{~dB}$ \\
Pass & $4.4 \mathrm{~dB}$ & $4.4 \mathrm{~dB}$ & $4.4 \mathrm{~dB}$ \\
Drop & $2.3 \mathrm{~dB}$ & $1.9 \mathrm{~dB}$ & $1.9 \mathrm{~dB}$ \\
\hline \hline
\end{tabular}

signals emitted from the access network to the backbone traffic. The operation mode is as follows:

1) Drop: A signal reaches the OADM through the input port. Then, a pair of quantum and conventional CWDM channels are filtered via two one-channel CWDM filters, $F_{q}$ and $F_{c}$, respectively, centered at the appropriate wavelength. Both are routed using two circulators and later multiplexed by a $1310 /$ 1550 WDM mux. Finally, the multiplexed signal leaves the backbone node through the add/drop (access network) port.

2) Pass: A signal reaches the OADM through the input port. Except for a pair of quantum and conventional CWDM channels, which are filtered, the rest of the signal crosses two CWDM filters, $F_{q}$ and $F_{c}$. The signal is later combined with the one coming from the entanglement source and the access network. Thus, if the input signal has no $F_{q}$ nor $F_{c}$ components, it leaves the OADM through the exit port unaltered.

3) Add: A signal reaches the OADM through the add/drop port (access network). It is then demultiplexed into quantum and conventional bands by a 1310/1550 WDM mux. Both bands are routed using two circulators to another 1310/1550 WDM mux that joins them again. At this point, a couple of $1 \times 2$ splitters combines this signal with the output by the entanglement source $(S)$, and with the signal reflected by the CWDM filters $F_{q}$ and $F_{c}$. The combined signal leaves the OADM through the output port. The signal output by the OADM has then three origins: backbone, access network, and entanglement source.

Note that, if the backbone node does not have a source $S$, one of the splitters can be removed.

Table II shows the losses of the node, calculated using the values from Table I. Clearly, the splitter is the component that has a larger impact on the overall losses. Nevertheless, we put the splitter from the source in the add path to minimize its effect: pass trough and drop signals are not affected. In a communication, a signal will suffer only once these extra losses, no matter 


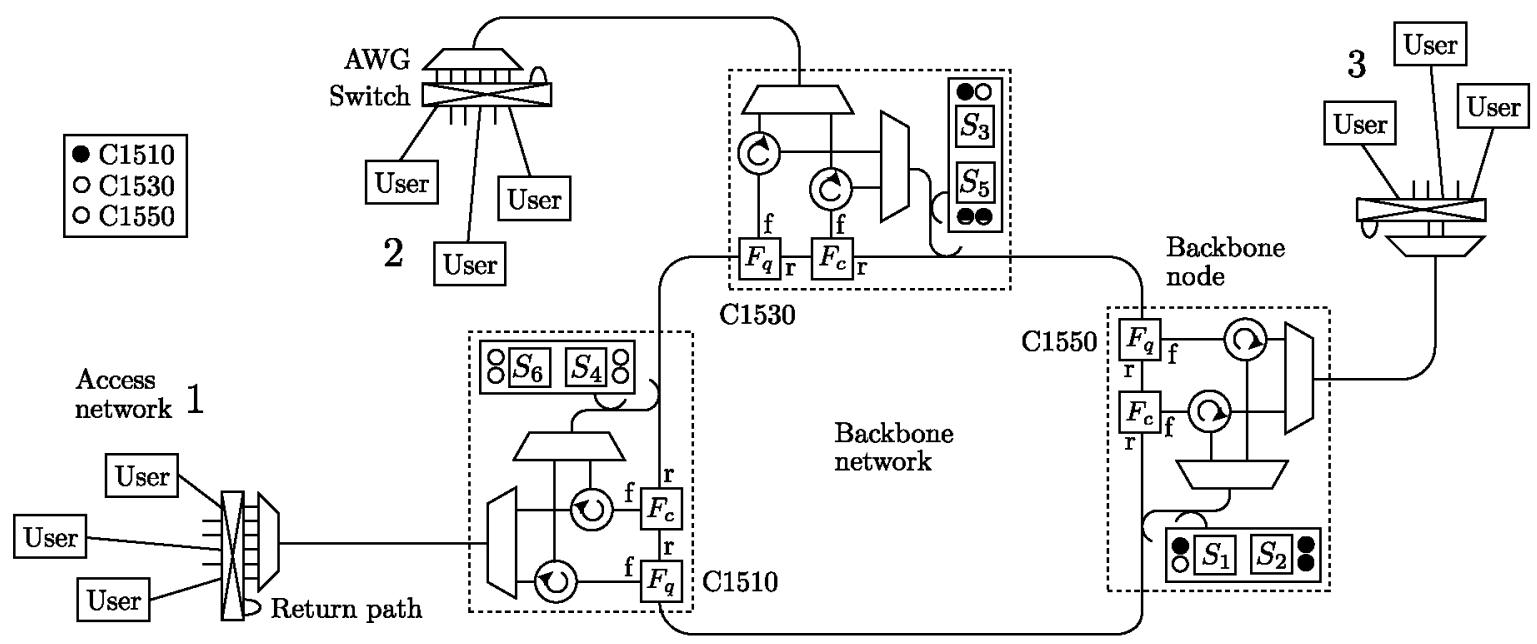

Fig. 9. Quantum MON based on the design shown in Section III. Besides allowing one-way communications, quantum and classical, the network is also capable of distributing entangled-photon pairs among any pair of users of the network. The pairs of circles represent the entangled photon-pairs produced by each source

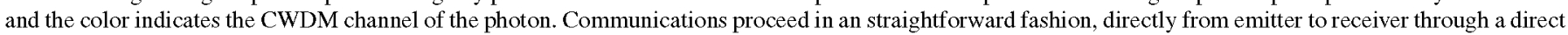
optical path, by using the channel plan depicted in Fig. 7. The selection of a specific wavelength by the emitter univocally determines its destination.

how far the destination is. Likewise, we use an unbalanced splitter not to hinder the signals coming from the access network (only $+0.5 \mathrm{~dB}$ for a $10 / 90$ splitter). Meanwhile, the increment of losses at the source's path $(11.3 \mathrm{~dB})$ can be counteracted by increasing the pumping power of the laser. Therefore, these losses are not taken into account when calculating the losses for adding a signal from the entanglement source.

\section{B. Access Network}

The current configuration of the access network routes all upstream signals to the backbone network. If two users from the same access network want to communicate directly, their signals will cross the entire backbone before reaching the receiver. This inefficient operation mode can be decisively improved by creating a short path within the access network. We propose to use a larger switch than needed and to use the extra ports to create loops in the network's side of the switch, i.e., return paths. Therefore, two users can connect to the user's side of the switch and use the loop to communicate directly. This is a simple, cost-free and local solution that does not introduce extra losses or modify the channel plan.

\section{Network Design}

As an example, we build a quantum MON using the described modifications. The network, depicted in Fig. 9, has three access networks $\left(A_{x}\right)$ and a backbone ring. The conventional and quantum CWDM channels assigned to each access networks are: (C1290, C1510) for $A_{1},(\mathrm{C} 1310, \mathrm{C} 1530)$ for $A_{2}$, and (C1330, $\mathrm{C} 1550$ ) for $A_{3}$. The entanglement sources $\left(S_{x}\right)$ are configured as in Fig. 7:

- $S_{1}$ serves $A_{1}(\mathrm{C} 1510)$ and $A_{2}(\mathrm{C} 1530)$ with $\lambda_{c}=1520 \mathrm{~nm}$

- $S_{2}$ serves only $A_{1}$ (C1510) with $\lambda_{c}=1510 \mathrm{~nm}$

- $S_{3}$ serves $A_{1}$ (C1510) and $A_{3}(1550)$ with $\lambda_{c}=1530 \mathrm{~nm}$

- $S_{4}$ serves only $A_{2}(\mathrm{C} 1530)$ with $\lambda_{c}=1530 \mathrm{~nm}$

- $S_{5}$ serves only $A_{3}(\mathrm{C} 1550)$ with $\lambda_{c}=1550 \mathrm{~nm}$

- $S_{6}$ serves $A_{2} \quad(\mathrm{C} 1530)$ and $A_{3} \quad(\mathrm{C} 1550)$ with $\lambda_{c}=1540 \mathrm{~nm}$.
TABLE III

PATH LoSSES From AN EMITTER (USER OR SOURCE) IN A QKD-MON WITH A FIXED-RING BACKBONE (SEE FIG. 9)

\begin{tabular}{lccc}
\hline \hline Path to & Losses Conv. & Losses Quant. & Losses Ent. \\
\hline 0-closest access network & $2.64 \mathrm{~dB}$ & $2.4 \mathrm{~dB}$ & - \\
One-closest access network & $20.36 \mathrm{~dB}$ & $18.4 \mathrm{~dB}$ & $11.2 \mathrm{~dB}$ \\
Two-closest access network & $26.04 \mathrm{~dB}$ & $23.6 \mathrm{~dB}$ & $16.4 \mathrm{~dB}$ \\
Three-closest access network & $31.72 \mathrm{~dB}$ & $28.8 \mathrm{~dB}$ & $21.6 \mathrm{~dB}$ \\
\hline \hline
\end{tabular}

Values calculated using Tables I and II.

This arrangement is represented in the figure using colored circles located near each source. They represent the entangled photon-pairs generated by the source. The color indicates the CWDM channel of the photon (i.e., the destination): blue for $\mathrm{C} 1510$, green for $\mathrm{C} 1530$, and orange for $\mathrm{C} 1550$. Note that the sources are deployed in a way that they always distribute photonpairs among the next and second next access networks in order to use the shortest paths.

We calculate the path losses in Table III using the considerations from Section III, and values from Tables I and II. We use the notation $x$-closest to denote proximity: 0-closest would be the same access network, one-closest the immediate next in the backbone direction, two-closest the immediate next after the one-closest, etc. Note that, in this design, there is no shortpath between an entanglement source and the immediate access network. Therefore, in order to reach the 0-closest access network, the signal has to travel the entire backbone network. For instance, in Fig. 9, the three-closest access network is actually the 0-closest, since, after crossing two backbone nodes, you are back to the immediate access network.

As shown, with a tolerable loss budget of $30 \mathrm{~dB}$, our network design allows one-way communications between nonneighboring access networks, e.g., from $A_{1}$ to $A_{3}$. For entangled communications, the longest path is between a neighboring access network and the next one, e.g., from $S_{2}$ to $A_{1}$ and $A_{2}$. The result is slightly worse in this case because the source 


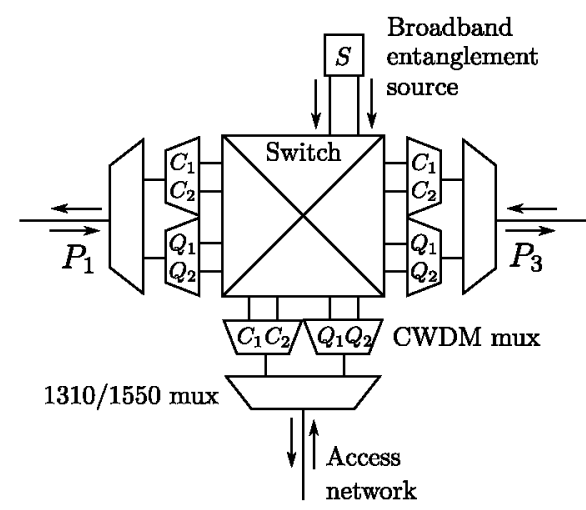

Fig. 10. Design of an active backbone node for a mesh-based backbone that includes a broadband source of entangled photon-pairs. Incoming signals are demultiplexed into quantum and conventional bands, and then into CWDM channels. These are routed to their corresponding port via a switch. All signals are multiplexed again before leaving the node.

always communicates with farther access networks. In the case of one-way, the source is already in one of the ends.

We estimate the maximum number of users ${ }^{5}$ following the same procedure as before. The first limitation comes from the losses. With a tolerable loss budget of $30 \mathrm{~dB}$, the network design is limited to three access networks (approximately 48 users). Adding a fourth access network would inevitably require a source to distribute pairs among non-neighboring access networks (e.g., $A_{2}$ and $A_{4}$ ) and to have one-way communications between three-closest access networks. Beyond the losses, we face again the limits from the spectral width of the source and then the number of CWDM channels available.

\section{Network Design Based on a Mesh-Shaped ACTIVE BACKBONE}

The network proposed in the previous section fulfills the intended goals, but falls short when facing a considerable increase in the number of users. The objective now is to devise a quantum MON using current technology able to grow and cope with a considerably larger number of users. For this, we conserve the discussed channel plan and access networks, but modify the backbone architecture using reconfigurable nodes and a mesh topology. This will reduce the losses and ensure architecture flexibility at the expense of losing the simplicity, reliability and low cost of the purely passive backbone design in the previous section.

\section{A. Backbone Node}

An active and reconfigurable version of the backbone node is shown in Fig. 10. The design is a transparent optical crossconnect, also referred to as photonic cross-connect (PXC), adapted to our needs. The PXC allows to route any input signal to any output port (even the original one). This opens the

\footnotetext{
${ }^{5}$ Here we refer to the maximum number of users that could in principle communicate simultaneously. In practice, this is limited by the noise produced by conventional signals.
}

TABLE IV

Losses of the Active Backbone Node With ENTANGLEMENT-CAPabILITY DePICTED IN FIG. 10

\begin{tabular}{lccc}
\hline \hline Action & Losses Conv. & Losses Quantum & Losses Ent. \\
\hline Cross & $4 \mathrm{~dB}$ & $4 \mathrm{~dB}$ & $2.5 \mathrm{~dB}$ \\
\hline \hline
\end{tabular}

door to use a dynamic assignment of CWDM channels for the access networks. The operation mode is simplified to only one function:

1) Cross: Signals reach the PXC through a port and they are separated into quantum and conventional bands by a $1310 /$ 1550 WDM mux. Both bands are demultiplexed into CWDM channels and sent to the switch. The switch routes each channel to the corresponding CWDM mux. CWDM channels are again multiplexed and combined into one signal that leaves the PXC.

In case that entanglement sources are needed, they are directly connected to the switch.

Note that due to the new topology, the backbone node uses the wavelength of the signal to decide through which output port the signal goes. Previously, the wavelength was used to decide whether to drop part of the signal or not. Hence, CWDM channels are now assigned to the communication paths instead of to the receivers.

In comparison with the OADM, the losses of the PXC are slightly lower due to the absence of splitters (see Table IV). However, this depends on the number of CWDM channels used per band. In our calculations, we have considered 4 , which is almost the worst-case scenario due to the width of the quantum and conventional bands (approximately $100 \mathrm{~nm}$ ). Although the figure shows a big switch for all signals, we can use separate switches for each of them without increasing the losses.

We highlight the fact that the losses of the node are independent of its degree (number of ports). Signals will still cross the same number of components. This is really helpful when dealing with dense areas like metropolitan ones. For example, we can create redundant paths between nodes for resiliency. Another interesting use case is to connect more than one access network per node. We increase the number of users per area but without adding more backbone nodes.

\section{B. Network Design}

Fig. 11 depicts a quantum MON based on active backbone nodes, a mesh topology, and 4 access networks $\left(A_{1}, A_{2}, A_{3}, A_{4}\right)$. Even though the network has more users and access networks, it only uses four sources and two CWDM channels per band: C1290 and C1310, and C1530 and C1550, for conventional and quantum signals, respectively. The communication scheme is still any-to-any, but not simultaneously. CWDM channels are not fixedly mapped to any access network. Furthermore, using less CWDM channels allows to reduce the number of different sources. Instead of having different sources, we configure the PXCs to route their signal to different access networks.

In contrast to the previous network design, the backbone nodes have to be configured carefully. We have to enable direct 


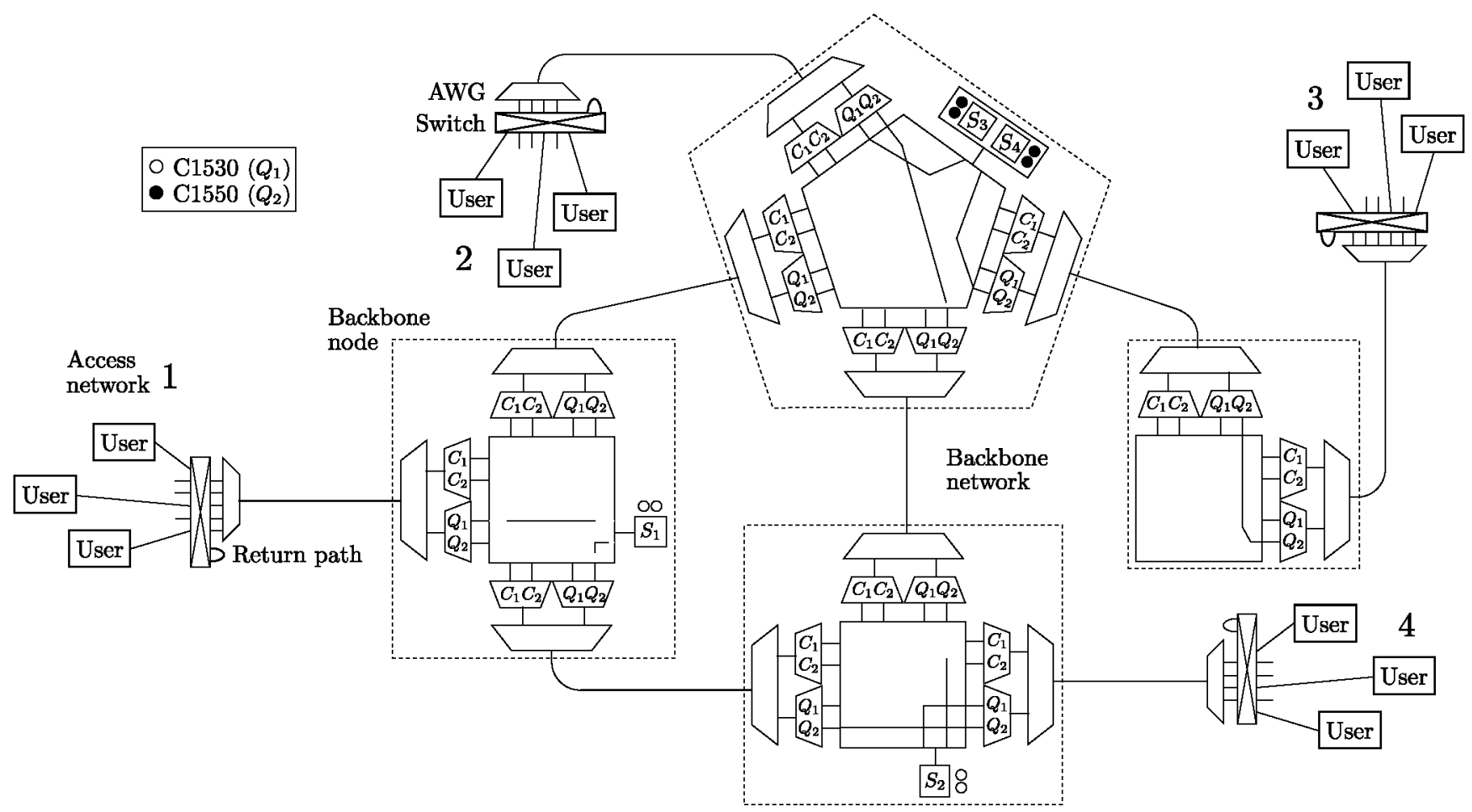

Fig. 11. Quantum MON with a mesh-type backbone, backbone nodes based on active technology, and four access networks ( $A_{x}$ ). Reconfiguring the nodes allows to interconnect all users using only two CWDM channels for quantum signals, but not at the same time. In the depicted configuration, entanglement is shared between $A_{1}$ and $A_{4}, A_{2}$ and $A_{4}, A_{2}$ and $A_{3}$, and within $A_{3}$. One-way, quantum and classical communication paths are not configured.

optical paths and entanglement-distribution between any pair of users using a fixed number of CWDM channels in the minimum number of steps. For instance, considering the networks in the figure, we need to create the following optical paths a total of 12 pairs:

- Enable direct optical paths between: $\left(A_{1}, A_{2}\right),\left(A_{1}, A_{3}\right)$, $\left(A_{1}, A_{4}\right),\left(A_{2}, A_{3}\right),\left(A_{2}, A_{4}\right)$ and $\left(A_{3}, A_{4}\right)$. These are bidirectional, thus $\left(A_{1}, A_{4}\right)$ is physically equal to $\left(A_{4}\right.$, $\left.A_{1}\right)$. Moreover, we do not need to address direct paths between users from the same access network since that is already solved by the return paths at the switch.

- Distribute entanglement between: $\left(A_{1}, A_{1}\right),\left(A_{1}, A_{2}\right),\left(A_{1}\right.$, $\left.A_{3}\right),\left(A_{1}, A_{4}\right),\left(A_{2}, A_{2}\right),\left(A_{2}, A_{3}\right),\left(A_{2}, A_{4}\right),\left(A_{3}, A_{3}\right)$, $\left(A_{3}, A_{4}\right)$ and $\left(A_{4}, A_{4}\right)$, covering thus any pair of users.

The problem is rather trivial as long as we have enough sources and loss budget. As an example, we show a series of network configurations in Fig. 12 for the network depicted in Fig. 11. The figure details each configuration over a simplified version of the network. The following three configurations cover all possible communications between pairs of access networks:

- Configuration 1: entanglement distribution to the pairs $\left(A_{1}\right.$, $\left.A_{2}\right)$ and $\left(A_{2}, A_{3}\right)$, and direct optical paths between the pairs $\left(A_{1}, A_{4}\right)$ and $\left(A_{3}, A_{4}\right)$.

- Configuration 2: entanglement distribution to $\left(A_{1}, A_{3}\right)$ and $\left(A_{1}, A_{4}\right)$, and direct optical paths between $\left(A_{2}, A_{4}\right)$ and $\left(A_{2}, A_{3}\right)$.

- Configuration 3: entanglement distribution to $\left(A_{2}, A_{4}\right)$ and $\left(A_{3}, A_{4}\right)$, and direct optical paths between $\left(A_{1}, A_{2}\right)$ and $\left(A_{1}, A_{3}\right)$.
Similarly, the network can be configured also to distribute entanglement to users from the same access network. Then, we can choose to use a fixed set of configurations that cover all possible communication paths and just reuse them over time, or actively switch the configurations depending on the user traffic at the moment.

We recalculate the path losses in Table V. As expected, the values are lower because the backbone node introduces less losses. Moreover, entanglement sources can now communicate with receivers located at the immediate access network, i.e., the access network that is connected to the same backbone node as the source. Keeping in mind the $30 \mathrm{~dB}$ tolerable loss budget for $\mathrm{QKD}$, the new design is limited to quantum communications between access networks separated by two intermediate backbone nodes: one way, $28.2 \mathrm{~dB}$, and entangled, $29.2 \mathrm{~dB}(12.2+17)$.

Besides allowing communications among farther locations, the number of users is also no longer a problem. In terms of loss budget, the mesh topology allows to add access networks without surpassing the worst-case path (in terms of losses) of the network by adding links that bypass intermediate backbone nodes. On the other hand, using PXCs and a dynamic assignment of CWDM channels allows to change the assignment of CWDM channels if we have more access networks than CWDM channels. Hence, the spectrum width of the source is not a limitation any more. Finally, even if all available CWDM channels are used, the mix of active nodes plus mesh topology allows to reuse them in different parts of the network at the same time (see Fig. 12). 


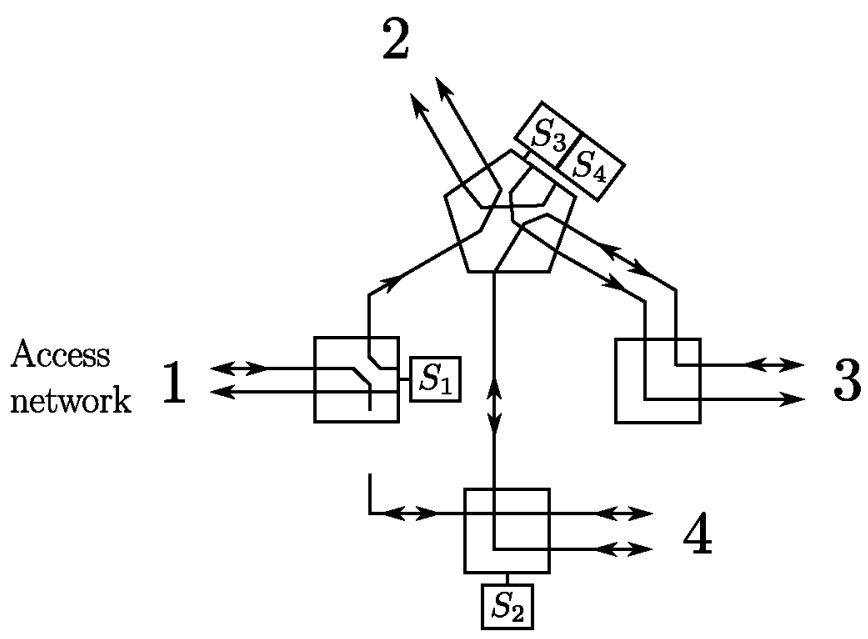

(a)

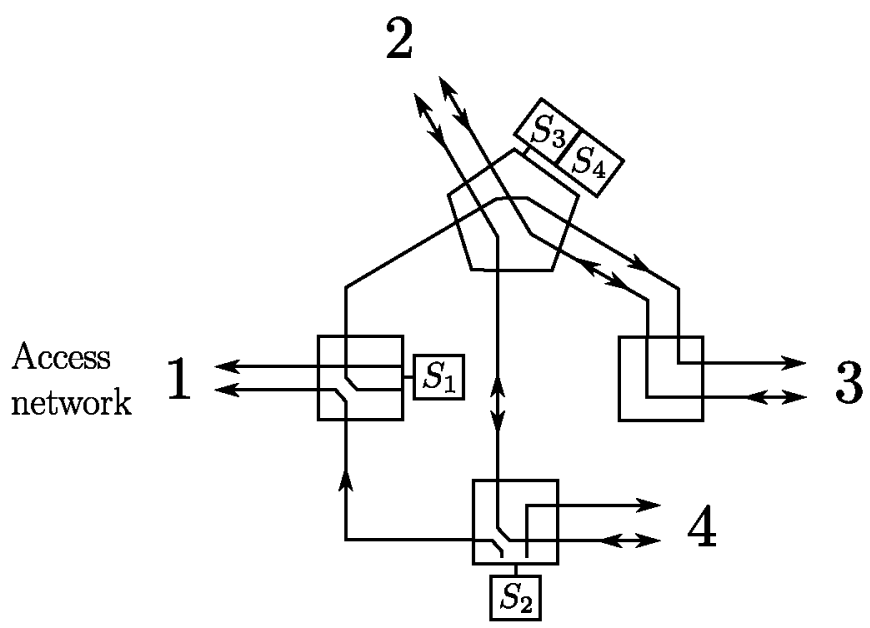

(b)

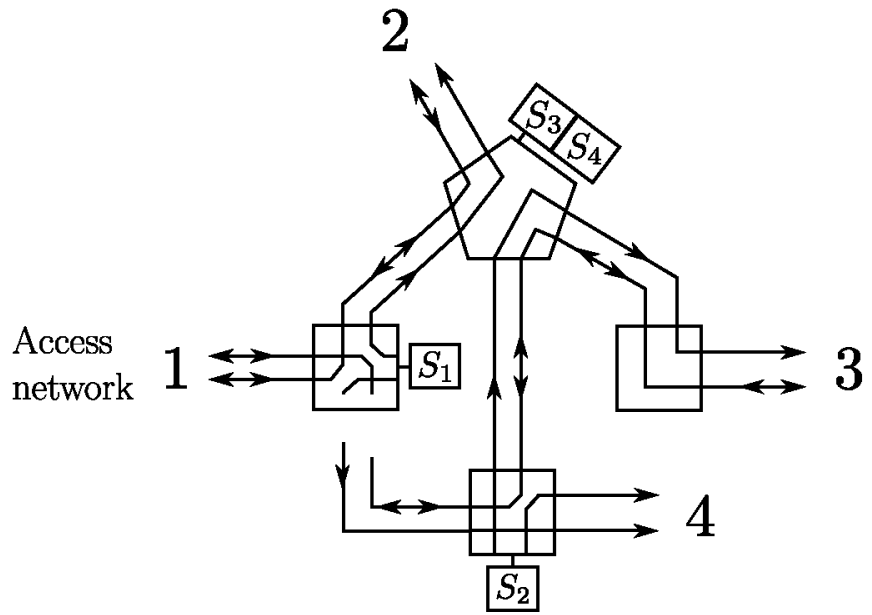

(c)

Fig. 12. Possible node configurations of the quantum MON shown in Fig. 11. Each backbone node is depicted as an schematic switch-box, and each color represents a CWDM channel for quantum signals. The three configurations cover all communication paths between pairs of access networks. (a) Configuration 1. (b) Configuration 2. (c) Configuration 3.
TABLE V

Path Losses From an EMITter (USER or SOURCE) IN A QKD-MON With a Reconfigurable-Mesh Backbone (SEe Fig. 11)

\begin{tabular}{lccc}
\hline \hline Path to & Losses Conv. & Losses Quant. & Losses Ent. \\
\hline O-closest access network & $2.64 \mathrm{~dB}$ & $2.4 \mathrm{~dB}$ & $7.4 \mathrm{~dB}$ \\
One-closest access network & $20.16 \mathrm{~dB}$ & $18.6 \mathrm{~dB}$ & $12.2 \mathrm{~dB}$ \\
Two-closest access network & $25.44 \mathrm{~dB}$ & $23.4 \mathrm{~dB}$ & $17 \mathrm{~dB}$ \\
Three-closest access network & $30.72 \mathrm{~dB}$ & $28.2 \mathrm{~dB}$ & $21.8 \mathrm{~dB}$ \\
\hline \hline
\end{tabular}

Values calculated using Tables I and IV.

In the end, the network can grow and the only effect will be an increase in the number of configurations needed to cover all possible communication paths. It is equivalent to decomposing the reconfigurable network into smaller fixed ones.

\section{DISCUSSION}

In this paper we have proposed several optical network designs that allow the distribution of entangled photon-pairs alongside with one-way quantum and conventional communications. This is especially helpful if we want to embrace as many quantum information technologies as possible and also to share the costs of the infrastructure.

Starting from a single access network, first we have shown a simple MON dedicated only to the distribution of entangled photon-pairs. It requires few components, which reduces the costs and the losses. The network is based on a mixture of coarse and dense wavelength multiplexing and can serve up to eight access networks (approximately 128 users using a $100 \mathrm{GHz}$ DWDM grid). Nevertheless, entanglement-based protocols and other quantum information technologies typically require also a direct optical path for quantum and conventional communications. For this task, we have proposed a channel plan that includes all these signals and two different network designs.

The first complete network design that includes all the signals is based on a backbone with ring topology and fixed passive nodes (OADMs). It is limited to three access networks with current quantum technology (approximately 48 users). In order to go beyond this network size, we have explored a more flexible network architecture based on a backbone with mesh topology and reconfigurable active nodes (PXCs).

The new design is not limited in terms of users. Moreover, with the same tolerable loss budget, it permits farther communications. The flexibility of using at will different paths to reach the same destination also makes the network resilient to attacks and link failures although the passive components used in the previous design are more robust and reliable. Nevertheless, these benefits come at a price. First, both the deployment and operational costs increase considerably. Switches are not, in general, a cheap component (depending on the number of ports) and active nodes require conditioned facilities, a constant supply of energy, more maintenance and a management protocol. Moreover, the management layer becomes more complex as we add nodes that, in the end, can compromise the scalability. Adding or removing backbone nodes, links and/or access networks in this case requires a complete reconfiguration. 


\section{REFERENCES}

[1] C. H. Bennett, G. Brassard, and N. D. Mermin, "Quantum cryptography without Bell's theorem," Phys. Rev. Lett., vol. 68, no. 5, pp. 557-559, 1992.

[2] C. H. Bennett and S. J. Wiesner, "Communication via one- and twoparticle operators on Einstein-Podolsky-Rosen states," Phys. Rev. Lett., vol. 69 , no. 20 , pp. 2881-2884, 1992.

[3] C. H. Bennett, G. Brassard, C. Crépeau, R. Jozsa, P. Asher, and W. K. Wootters, "Teleporting an unknown quantum state via dual classical and Einstein-Podolsky-Rosen channels," Phys. Rev. Lett., vol. 70, no. 13, pp. 1895-1899, 1993.

[4] P. D. Townsend, "Simultaneous quantum cryptographic key distribution and conventional data transmission over installed fibre using wavelengthdivision multiplexing," Electron. Lett., vol. 33, no. 3, pp. 188-190, 1997.

[5] N. A. Peters, P. Toliver, T. E. Chapuran, R. J. Runser, S. R. McNown, C. G. Peterson, D. R. Rosenberg, N. Dallmann, R. J. Hughes, K. P. McCabe, J. E. Nordholt, and K. T. Tyagi, "Dense wavelength multiplexing of $1550 \mathrm{~nm}$ QKD with strong classical channels in reconfigurable networking environments," New J. Phys., vol. 11, no. 4, p. 045012, 2009.

[6] P. Eraerds, N. Walenta, M. Legré, N. Gisin, and H. Zbinden, "Quantum key distribution and 1 Gbps data encryption over a single fibre," New $J$. Phys., vol. 12, no. 6, p. 063027, 2010.

[7] B. Qi, W. Zhu, L. Qian, and H.-K. Lo, "Feasibility of quantum key distribution through a dense wavelength division multiplexing network," $\mathrm{New}$ J. Phys., vol. 12, no. 10, p. 103042, 2010.

[8] K. A. Patel, J. F. Dynes, M. Lucamarini, I. Choi, A. W. Sharpe, Z. L. Yuan, R. V. Penty, and A. J. Shields, "Quantum key distribution for $10 \mathrm{~Gb} / \mathrm{s}$ dense wavelength division multiplexing networks," Appl. Phys. Lett., vol. 104, no. 5, pp. 051123-1-051123-4, 2014.

[9] R. J. Hughes, G. G. Luther, G. L. Morgan, C. G. Peterson, and C. Simmons, "Quantum cryptography over underground optical fibers," in Proc. Adv. Cryptol. Conf., 1996, pp. 329-342.

[10] C. Holloway, E. Meyer-Scott, C. Erven, and T. Jennewein, "Quantum entanglement distribution with $810 \mathrm{~nm}$ photons through active telecommunication fibers," Opt. Exp., vol. 19, no. 21, pp. 20597-20603, 2011.

[11] J. F. Dynes, I. Choi, A. W. Sharpe, A. R. Dixon, Z. L. Yuan, M. Fujiwara, M. Sasaki, and A. J. Shields, "Stability of high bit rate quantum key distribution on installed fiber," Opt. Exp., vol. 20, no. 15, pp. 16339-16347, 2012.

[12] K. Shimizu, T. Honjo, M. Fujiwara, T. Ito, K. Tamaki, S. Miki, T. Yamashita, H. Terai, Z. Wang, and M. Sasaki, "Performance of longdistance quantum key distribution over $90-\mathrm{km}$ optical links installed in a field environment of tokyo metropolitan area," J. Lightw. Technol., vol. 32, no. 1, pp. 141-151, Jan. 2014.

[13] I. Choi, Y. R. Thou, J. F. Dynes, Z. Yuan, A. Klar, A. Sharpe, A. Plews, M. Lucamarini, C. Radig, J. Neubert, H. Griesser, M. Eiselt, C. Chunnilall, G. Lepert, A. Sinclair, J.-P. Elbers, A. Lord, and A. Shields, "Field trial of a quantum secured $10 \mathrm{~Gb} / \mathrm{s}$ DWDM transmission system over a single installed fiber," Opt. Exp., vol. 22, no. 19, pp. 23121-23128, 2014.

[14] D. Lancho, J. Martinez, D. Elkouss, M. Soto, and V. Martin, "QKD in standard optical telecommunications networks," Lecture Notes Inst. Comput. Sci., Soc. Informat. Telecommun. Eng., vol. 36, no. 11, 2010, pp. 142-149.

[15] J. Capmany and C. R. Fernandez-Pousa, "Optimum design for BB84 quantum key distribution in tree-type passive optical networks," J. Opt. Soc. Amer. B, vol. 27, no. 6, pp. A146-A151, 2010.

[16] I. Choi, R. Young, and P. D. Townsend, "Quantum information to the home," New J. Phys, vol. 13, no. 6, p. 063039, 2011.

[17] M. Razavi, "Multiple-access quantum key distribution networks," IEEE Trans. Commun., vol. 60, no. 10, pp. 3071-3079, Oct. 2012.

[18] B. Fröhlich, J. F. Dynes, M. Lucamarini, A. W. Sharpe, Z. Yuan, and A. J. Shields, "A quantum access network," Nature, vol. 501, no. 7465 , pp. 69-72, 2014.

[19] S. Aleksic, F. Hipp, A. Poppe, B. Schrenk, D. Winkler, and G. Franzl, "Towards a smooth integration of quantum key distribution in metro networks," presented at the 16th Int. Conf. Transp. Opt. Netw., Graz, Austria, 2014, Paper Tu.B1.1.

[20] J. Martinez-Mateo, A. Ciurana, and V. Martin, "Quantum key distribution based on selective post-processing in passive optical networks," IEEE Photon. Technol. Lett., vol. 26, no. 9, pp. 881-884, May. 2014

[21] I. Choi, R. Young, and P. D. Townsend, "Quantum key distribution on a 10 Gb/s WDM-PON," Opt. Exp., vol. 18, no. 9, pp. 9600-9612, 2010.

[22] G. Brassard, F. Bussieres, N. Godbout, and S. Lacroix, "Entanglement and Wavelength division multiplexing for quantum cryptography networks," in Proc. AIP Conf., 2004, vol. 734, no. 1, pp. 323-326.
[23] H. C. Lim, A. Yoshizawa, H. Tsuchida, and K. Kikuchi, "Wavelengthmultiplexed entanglement distribution," Opt. Fiber Technol., vol. 16, no. 4 , pp. $225-235,2010$.

[24] J. Ghalbouni, I. Agha, R. Frey, E. Diamanti, and I. Zaquine, "Experimental wavelength-division-multiplexed photon-pair distribution," Opt. Lett., vol. 38 , no. 1 , pp. 34-36, 2013.

[25] I. Herbauts, B. Blauensteiner, A. Poppe, T. Jennewein, and H. Hübel, "Demonstration of active routing of entanglement in a multi-user network," Opt. Exp., vol. 21, no. 23, pp. 29013-29024, 2013.

[26] F. Xu, W. Chen, S. Wang, Z. Yin, Y. Zhang, Y. Liu, Z. Zhou, Y. Zhao, H. Li, D. Liu, Z. Han, and G. Guo, "Field experiment on a robust hierarchical metropolitan quantum cryptography network," Chin. Sci. Bull., vol. 54, no. 17, pp. 2991-2997, 2009.

[27] A. Ciurana, J. Martinez-Mateo, M. Peev, A. Poppe, N. Walenta, H. Zbinden, and V. Martin, "Quantum metropolitan optical network based on wavelength division multiplexing," Opt. Exp., vol. 22, no. 2, pp. 1576 1593,2014

[28] S. Wang, W. Chen, Z.-Q. Yin, Y. Zhang, T. Zhang, H.-W. Li, F.-X. Xu, Z. Zhou, Y. Yang, D.-J. Huang, L.-J. Zhang, F.-Y. Li, D. Liu, Y.-G. Wang, G.-C. Guo, and Z.-F. Han, "Field test of wavelength-saving quantum key distribution network," Opt. Lett., vol. 35, no. 14, pp. 2454-2456, 2010.

[29] S. Wang, W. Chen, Z.-Q. Yin, H.-W. Li, D.-Y. He, Y.-H. Li, Z. Zhou, X.-T. Song, F.-Y. Li, D. Wang, H. Chen, Y.-G. Han, J.-Z. Huang, J.-F. Guo, P.-L. Hao, M. Li, C.-M. Zhang, D. Liu, W.-Y. Liang, C.-H. Miao, P. Wu, G.-C. Guo, and Z.-F. Han, "Field and long-term demonstration of a wide area quantum key distribution network," Opt. Exp., vol. 22, no. 18, pp. 21739-21756, 2014.

[30] A. Treiber, A. Poppe, M. Hentschel, D. Ferrini, T. Lorünser, E. Querasser T. Matyus, H. Hübel, and A. Zeilinger, "A fully automated entanglementbased quantum cryptography system for telecom fiber networks," $N e w J$. Phys., vol. 11, no. 4, p. 045013, 2009.

[31] T. Lorunser, A. Happe, and A. Poppe, "Timing synchronization with photon pairs for quantum communications," presented at the Lasers ElectroOpt. Eur. Int. Quantum Electron. Conf., Munich, Germany, 2013.

[32] M. B. Nasr, S. Carrasco, B. E. A. Saleh, A. V. Sergienko, M. C. Teich, J. P. Torres, L. Torner, D. S. Hum, and M. M. Fejer, "Ultrabroadband biphotons generated via chirped quasi-phase-matched optical parametric down-conversion," Phys. Rev. Lett., vol. 100, p. 183601, 2008.

[33] B. Blauensteiner, I. Herbauts, S. Bettelli, A. Poppe, and H. Hübel, "Photon bunching in parametric down-conversion with continuous-wave excitation," Phys. Rev. A, vol. 79, p. 063846, 2009.

[34] R. Ramaswami, K. Sivarajan, and G. Sasaki, Optical Networks: A Practical Perspective,. 3rd ed. San Mateo, CA, USA: Morgan Kaufmann, 2009.

[35] Spectral Grids for WDM Applications: CWDM Frequency Grid, ITU-T Standard G.694.2, 2003.

[36] Spectral Grids for WDM Applications: DWDM Frequency Grid, ITU-T Standard G.694.1, 2002.

[37] Corning: SMF-28e+ LL optical fiber. [Online]. Available: http://www. corning.com/

[38] Flyin Optronics: splitter, circulators, CWDM and DWDM filters, and 1310/1550 WDM multiplexers. [Online]. Available: http://www. flyinoptronics.com/

[39] Polatis: optical switch Series 6000. [Online]. Available: http://www. polatis.com/products/

[40] LG Nortel WPF $1132 \mathrm{C}$ (32-channels AWG).

[41] W. Maeda, A. Tanaka, S. Takahashi, A. Tajima, and A. Tomita, "Technologies for quantum key distribution networks integrated with optical communication networks," IEEE J. Sel. Topics Quantum Electron., vol. 15, no. 6, pp. 1591-1601, Nov./Dec. 2009

[42] T. E. Chapuran, P. Toliver, N. A. Peters, J. Jackel, M. S. Goodman, R. J. Runser, S. R. McNown, N. Dallmann, R. J. Hughes, K. P. McCabe, J. E. Nordholt, C. G. Peterson, K. T. Tyagi, L. Mercer, and H. Dardy, "Optical networking for quantum key distribution and quantum communications," New J. Phys, vol. 11, no. 10, p. 105001, 2009. 